\title{
Advances in neural network based learning
}

\author{
Xi-Zhao Wang • Abdallah Bashir Musa
}

Received: 17 November 2013/ Accepted: 21 November 2013/Published online: 5 December 2013

(C) Springer-Verlag Berlin Heidelberg 2013

In recent years, the applications of diverse machine leaning algorithms and their fusion to the cybernetics and decisionmaking have been attracting more and more scholars from different disciplines. As a most frequently used technique of extracting knowledge from data, a feed-forwarded neural network (NN) has shown more advantages for the regression and classification problems. The main advantages come from the capability of good approximation of NNs and their corresponding highly nonlinear boundary. Due to the many merits and wide applications of NNs, the study on several fundamental issues of NNs, such as structure selection, overtraining, robustness and capability of resistance to noisy data, training on different types of data, and more importantly, the generalization ability, are still in progress although NNs have been an old topic in the areas of learning and reasoning. As a result, these extensive studies and significant improvements bring a number of new features anddevelopments to NNs. This issue makes an attempt to provide some latest advances of $\mathrm{NN}$ learning, the recent improvements of performance for NN learning systems, and new applications of NNs to different real fields.

In this issue, 14 papers are accepted for publication. The 14 papers cover a variety of topics which include the stochastic stability NN with time delay, imbalanced and uncertain data, fuzzy NNs, bidirectional associative memory (BAM), deep learning of NNs, and other comparative

\footnotetext{
X.-Z. Wang · A. B. Musa ( $ه)$

Key Laboratory in Machine Learning and Computational Intelligence of Hebei Province, College of Mathematics and Computer Science, Hebei University, Baoding 071002, China

e-mail: abdubashir20@yahoo.com

X.-Z. Wang

e-mail: xizhaowang@ieee.org
}

and review studies. Categorization and brief description of these papers are given below.

Four papers discuss the stability of NNs with delay time. Actually, the time delay is ubiquitous in most natural systems. Since the time delay is frequently encountered in NNs, the issue about stability of NNs with delay has been emerging as a challenging task for NN researchers. The paper authored by Cheng-De Zheng studied a class of stochastic reaction diffusion NNs with Markovian jumping parameters and time delays. Lyapunov functional method and stochastic analysis technique are used there to construct a new stochastic stability in the principle of the mean square. Continuing this topic, the paper authored by $\mathrm{M}$. Syed Ali studied the stochastic stability analysis of uncertain recurrent NNs, also with Markovian jumping parameters and time varying delays. By using Lyapunov functional theory, Itô differential rule and matrix analysis techniques, a sufficient criterion in term of linear matrix inequality (LMI) is established such that, for all admissible parameter uncertainties and stochastic disturbances, the stochastic NN is perfectly stable. And then, a non-stationary problem on BAM NNs is discussed in Qingqing He's paper. An exponential stability in terms of LMIs is achieved for a class of interval C-G type (BAM) NNs with the mixed time delays and non-smooth behaved functions by using Homomorphic mapping theory, non-smooth analysis, LMIs, free-weighting matrix and Lyapunov-krasovskii functional approach. Furthermore, the paper authored by R. Raja derived the stability analysis for a class of uncertain discrete-time stochastic BAM NNs with timevarying delays and impulses. Lyapunov-Krasovskii functional and stochastic analysis techniques were applied to obtain a set of sufficient conditions in term of LMI.

Along this line, the non-stationary challenge in other class of NN can be tackled. The paper authored by Adel 
Ghazikhani discussed a problem of non-stationary and imbalanced data stream classification (DSC) in the online NNs. An online MLP classifier for non-stationary and imbalanced data streams was constructed where the nonstationary and imbalance were handled by using a forgetting function and a specific error function respectively. Another paper authored by Guohua Liang conducted an empirical study on the effects of different levels of imbalanced class distribution regarding the bagging predictors by using the under-sampling techniques.

The modification to existing algorithms or construction of new algorithms to solve new problems in NN area is always definitely meaningful. For solving the problem of large saturation regions available in current activation functions such as Sigmoid, the paper authored by JinCheng Li developed a differentiable bi-firing activation function with a very small saturation region for deep neural networks (DNN). The paper authored by Huaiqin $\mathrm{Wu}$ discussed an inverse optimal value problem with convex constraint and then a form of nonlinear NNs is designed for solving this problem. The NNs and fuzzy NNs working as approximators for the discontinuous fuzzy functions were investigated in the paper authored by Chunmei He, who used a polygonal fuzzy NN as an approximator in the sense of Choquet integral norm with respect to the sub additive fuzzy measure.

For improving the learning from data with an instancevarying importance, the paper authored by Peter Sarlin represented a weighted self-organizing map (WSOM) that combines the advantages of the standard SOM paradigm with learning that accounts for instance-varying importance.
The paper authored by Gian Luca Marcialis proposed a novel method for automatic head-pose estimation based on a geometrical model relying on the exploitation of the Vitruvian man's proportions and the related Golden Ratio, where the simple ratios among eyes and nose positions are used to compute the pitch angle.

The comparative and review studies are considered as an essential part for any area of research because it can build an excellent foundation for the further works. In this issue, a comparative study among NN, least squares support vector machine (LSSVM) and a hybrid approach called APSO-LSSVM on the prediction of throughput in coal ports is conducted by Shuang Liu. Also, a comparison among radial basis function NNs, adaptive neuro-fuzzy inference systems (ANFIS), and genetically evolved fuzzy inference systems (G-FIS) on prediction in the end milling process is investigated by Abdel Badie Sharkawy. Finally, Chun-Wei Tsai conducted a review study on the smart grid by reviewing some fundamental issues from the perspective of computational awareness.

We hope that the different kinds of papers included in this issue can inform the interested readers and researchers some latest advances on NN based learning. Also we hope these selected papers are helpful for the readers to conduct the further studies on NN based learning, to develop the new learning systems based on NNs, and to explore the new real applications.

Thanks go to authors for their contributions to this issue, and also go to reviewers for their detailed comments and constructive suggestions of improving the submission quality. 